\title{
Homeostatic Conductance and Parasympathetic Basis Alteration: Two Alternative Approaches to Deep Brain Stimulation in Parkinson's, Obsessive Compulsive Disorder and Depression
}

\author{
Richard Lawrence Norman \\ Editorial Department, Journal of Unconscious Psychology and Mind Magazine, O'Brien, USA \\ Email: editor@thejournalofunconsciouspsychology.com
}

Received 19 January 2016; accepted 23 February 2016; published 26 February 2016

Copyright (C) 2016 by author and Scientific Research Publishing Inc.

This work is licensed under the Creative Commons Attribution International License (CC BY).

http://creativecommons.org/licenses/by/4.0/

(c) (i) Open Access

\begin{abstract}
Derived through the dual focus of psychology and neuroscience, disciplines such as Affective Neuroscience and Neuropsychoanalysis may offer new directions of approach to many pathologies, which demonstrate psychical and somatic etiology. Insights gained from these two trustworthy yet still peripheral scientific disciplines, are deserving of consideration and exploration. It is the purpose of this paper to offer up several such possible new avenues of exploration, as they relate to depression, Obsessive Compulsive Disorder (OCD), and Parkinson's Disease (PD). The basic therapeutic focus in deriving these proposed new clinical approaches is twofold: 1) Deep Brain Stimulation (DBS) and 2) Mnemic alteration via neuropeptide assisted therapy producing positive relative adjustment in the balance of basic sympathetic (dopaminergic) and parasympathetic affective circuitry independent of drugs or surgery. New conductance level and target strategies designed to prevent homeostatic degeneration are advanced under the concept of homeostatic conductance. New electrical stimulation targets are suggested, which restore basic rhythmic balance between pathologically affected and unaffected brain areas. In this way, we offer up new directions for research, strategies for noninvasive and nontoxic potential therapies and new targets/ approaches to DBS therapy.
\end{abstract}

\section{Keywords}

Deep-Brain-Stimulation, Obsessive-Compulsive-Disorder, Depression, Parkinson's, Hippocampus 


\section{Introduction}

New approaches to pathologies as diverse as depression, OCD and Parkinson's may be derived through study of the interactivity between psyche and soma. There is a great wealth of information and work which has been accrued detailing well known DBS approaches to Parkinson's [1]-[3] and more recently, depression and OCD [4]-[6]. However, longstanding falsehoods which have been deeply inculcated into scientific dogma, such as the incorrect myth that the dopaminergic systems of the Medial Forebrain-Bundle Lateral-Hypothalamic (MFB-LH) circuitry constitute a pleasure/reward center, have left basic flaws in scientific understanding [7] [8]. To properly understand the nuanced purposeful SEEKING system associated with the MFB-LH circuitry as a SEEKING system designed to solicit interest in engaging and exploring the world, rather than a simple pleasure center, is vital [7] [8]. There is a dearth of available specific neuroanatomical information relating to the dynamic systemic activational allocations corresponding to guilt, even though that information is more important than any other in addressing the causes of neurosis, psychosis and social control. As the reader may be aware, guilt and its close relation to repression, constitute the basis of human neurosis, as understood by depth psychology [9]. The absence of prolific neuroanatomical information relating to guilt is therefore deeply troubling. Neuropsychoanalysis [10] acknowledges the deep connectivity between specific brain processes, and proper depth psychology. Affective Neuroscience [7] provides a detailed and proper affective anatomical basis for human and animal subjects. Through study of the disciplines of affective neuroscience and neuropsychoanalysis, the missing information can be derived, and new vital directions for treatment advanced.

1) First we will introduce in a general way the most basic and fundamental sympathetic dopaminergic and parasympathetic noradrenergic socio-affective circuitry associated with the SEEKING system [7] [8], guilt and repression, which we propose in conditioned pro-parasympathetic allocational distribution constitute an internalized homeostatic imbalance, and hence, become a possible causal factor in the aforementioned disorders.

2) Next, we will detail two new approaches which offer potential reorientation of systemic functioning without adversely affecting homeostasis, so as to minimize homeostatic reactive degenerative deterioration. The first new approach is a manual rhythmic entrainment of pathologically affected areas based on those unaffected by degeneration. The second, is a restorative homeostatic approach which stimulates and places demand on the substantia nigra (SN) via efferent projections arising from the nucleus accumbens-septi. Two related secondary approaches (designated a. and b.) are also discussed.

3) Lastly, a more detailed extensive psychological and developmental circuit analysis will clarify a new noninvasive, non-toxic methodology, which may allow the partial amelioration of these diverse pathologies, as a function of hippocampal mnemic instantiation (and memory consolidation) assisted in its process plasticity via safe neuropeptide, altering internal dopaminergic distributions.

We assert: Following an affective psychologically contextualized model of human development and distributed anatomical allocation is vital in securing new directions of approach.

\section{Parasympathetic Pathological Genesis and Homeostatic Conductance}

Dopaminergic innervations projecting between the limbic and orbito-frontal regions are established as a product of neuroendocrine changes initiated via the dyadic exchange of (touch), gaze and glance shared by the mother and infant, forming the dopaminergically modulated sympathetic ventral tegmental limbic circuit within the first 18 months of life (Schore as cited in [10], p. 234). The complete primary basis regulatory socio-affective circuitry is structured in the addition of the socially conditioned inhibitory circuit: the noradrenergically modulated parasympathetic lateral limbic circuit (ibid. p. 235), which appears as a basis of repression proper, associated with social disapproval, shame and in later development guilt [11] [12]. We have deduced, that these two circuits in their relative attributional reactive balance constitute the fundamental affective basis of human personality, and have observed the associated creation and curtailment of Parkinsonian-like symptomatology, symptoms of OCD and depression as relative circuit balance is altered [12]. We believe after careful study and a-priori examination, that pro-parasympathetic/repressive imbalance between these two opposing circuits may well be to some degree causal in the aforementioned conditions. This is the meaning of parasympathetic pathological genesis. This parasympathetic pathological genesis is the parasympathetic basis of pathology to be curatively altered: hence the term parasympathetic basis alteration. For this reason, we propose it may be possible to ameliorate pathology associated with OCD, depression and Parkinson's by way of changing an element associated 
with pathologic genesis: by down-mediating the activations of the noradrenergically modulated parasympathetic lateral limbic circuit and/or up-mediating sympathetic dopaminergic expression.

Most approaches to DBS use high frequency electrical stimulation to gain effective results. We propose that the homeostatic basis of coordinated systemic functioning is thereby disrupted, and an approach which suits conductance levels and targets to the maintenance of homeostatic priority, may yield positive long lasting results, with less treatment induced systemic deterioration. This is the meaning of "homeostatic conductance". Conductance levels, targets and approaches for DBS should not disturb (and/or may reestablish) homeostasis and coordinated intrasystemic functioning. This is the principle of homeostatic conductance.

\section{Electro-Rhythmic Re-Entrainment, Homeostatic Conductance and DBS}

The mysterious fact that (bilateral) Deep Brain Stimulation (DBS) of the subthalamic nucleus, thalamus, or globus pallidus, which by the reasoning of cognitive neuroscience ([13], p. 305), should increase symptoms by way of thalamo-cortical inhibition, but does not, may in some small part be a product of activating the SEEKING system itself [7] [8] [14] which can typically be stimulated anywhere from the medial forebrain bundle-lateral hypothalamic circuitry, up to the nucleus accumbens to the medial prefrontal cortex via the mesolimbic and mesocortical dopamine pathways. It appears likely, that spontaneous activational inhibition and firing entrainment through a secondary artificial electro-rhythmic modulatory source functioning at the level of presynaptic activation may be at work. "Neurons close to the stimulation electrode had their spontaneous activity suppressed by activation of inhibitory presynaptic terminals, while their axons were directly activated. Axonal spike output thus became time-locked to the stimulus frequency" [15].

The Ventral Tegmental Area, unlike the Substantia Nigra, does not suffer degenerative necrosis in PD [16]. In Parkinson's, the Substantia Nigra (SN), and the Ventral Tegmental Area (VTA) become rhythmically desynchronized [17] [18]. We propose that dynamics causing low frequency oscillations and other symptoms in PD, may in some measure be resultant of this basic intra-systemic imbalance as proliferated through systemic expression. It may therefore be advantageous to manually electro-rhythmically re-entrain the SN and VTA with DBS. We may base the rhythmic entrainment DBS output projecting to the pathologically affected SN on the unaffected VTA patterning, and allocate a frequency specific pulsed electro-rhythmic "pace maker" output, designed to synchronize axonal firing, modeled on the pathologically unaffected VTA rhythm. Or, both areas could be rhythmically co-entrained to an external DBS source of electro-conductive distribution.

As stated above, we believe that there is a homeostatic factor such that feedback in the system has been a causally contributing dynamic element stemming from parasympathetic/repressive over-activity, and has all but "requested" the condition to emerge. So, if the dopaminergic motor contributions and those of the SEEKING system are stimulated with relatively intense electrical activity as it is now used in DBS regimens for Parkinson's, OCD and depression, then the system once aided, will re-balance to decay further. Instead, the progression of disease and degeneration may hypothetically be aided to resuscitation, or at least to end further degeneration, by another more subtle approach. We suggest several possibilities: The SEEKING (A-10) dopaminergic neurons project from the ventral tegmental area. If the system were stimulated here, or along the usual MFB-LH circuitry as in animal studies, or better still, at the superolateral branch of the medial forebrain bundle [19] and the stimulation were slight, not excessive, perhaps in cases of Parkinson's, over time without interfering with homeostasis, the demand placed upon the system, and hence demand placed upon the substantia nigra via conductance over efferent projections stemming from the nucleus accumbens-septi, would create a new systemic balance conducive to functional maintenance, or improvement.

a) Naturally occurring systemic intervention might be simulated with DBS. Along with therapy designed to lessen repressive psychological structuralization (see below), encouragement may be offered to the system. SEEKING activation is associated with heightened activity in the nucleus accumbens [7] [8]. Sexual excitation is a sure bet to engage the system. So, low level septal stimulation should be tried. Homeostatic conductance is key. Just enough to create a constant demand...the lowest amount to gain response in the nucleus accumbens.

b) A neuropeptide may be formulated so as to be deliverable to neural targets which activate the system... neurotensin for example ([7], p. 101). Or along the same lines...a dynorphin blocking agent, as dynorphins down-regulate SEEKING activation, an agent which blocks kappa opiate receptors may aid pathological amelioration ([8] er systemic balance may be encouraged, and resultant pathology lessened. 


\section{Hippocampal Plastic Mnemic Therapy: A Nonsurgical, Nontoxic, Theoretic Paradigm}

Pharmacology alters pathology by chemical influence of the electrochemical mediation of biological circuitry. DBS alters pathology by electro-conductive intervention altering the activity of biological circuitry. We wish to propose, that said circuitry may be altered to reduce pathology, by way of hippocampal plasticity, and mnemic instantiation. We will place this in the context of a condensed new theoretic structure [20].

The quality of experience, the hard problem of consciousness as it is known, might be understood rightly as a function of associative mnemic instantiation [21]-[23]. The following notion stems from a novel approach to restructuring past memories in cases of neurosis and abuse, which has proven itself effective in our private researches: Re-Polarization theory [23]. Although effective, re-polarization theory has shortcomings:

a) It requires the user to master a self-psychoanalytic technique which is psychically invasive.

b) Said technique is useful in cases of hysteria, but appears to be unavailable to many, specifically those who present with OCD due to high functioning repressive dynamism.

c) Said technique requires many deeply painful regressions to gain full efficacy.

d) Said technique requires the access of deeply repressed formative memories, so as to create regressions.

However, much can be gained by close examination and analysis of the functional end of re-polarization theory. These insights will serve us well as we tread this new pathway.

It will be helpful to create a working analogy, a schematic model for ego processes, which can be functionally ascribed to neuroanatomical coordinations extending systemic connectivity associated with the Default Mode Network [24]. Think of psychoanalysis and the role of ego as a stimulus barrier which mediates both internal and externally derived cathexis [9] (1923). We wish to suggest at this juncture the simple and correct idea that the combinative functionality of ego/super-ego in distributing and restricting the expression of unconscious affect, the forming of affective repressions and affective/libidinal distributions, may be usefully reduced to the idea of a non-commutative topographic experiential template. On the simplest level, imagine a stratified structure allocating resistance where height in vertical location corresponds to the strength of that element's dynamic contributions to the qualitative unconscious transference forming ontological reality. On a more nuanced level of analogy, please think of an associative neuronal attractor network, perhaps with epigenetic underpinnings, which defines perceived objects and situations - the attractor - in a nonlinear process of displaced affective valence akin to theories of quantum perception and collapse [21], where elements closer to the surface in the associative chain, those of greater energetic cathexis are greater contributors. Increased quanta of energetic cathexis, is akin in its effect, to raising the element up in the associative chain. [Think of quantitative energetic cathexis in terms of Freud's economic/quantitative factor [9] (1914-1916)]. The precise role of each typological participant in the formative unconscious transference must be quantitatively articulated if psychology is ever to claim its rightful place as hard science and place a real etiologically sound basis under this pluralistic discipline [22], and in addition, a clear relation must be established to phylogenetic epigenetics [25]-[28]. However, this simple starting point, will soon prove itself a useful gateway.

The designation non-commutative refers to the notion that the order of topographic assembly and relation is a functional contributor to manifest outcome. Ego often carries out the repressions of super-ego, and is therefore structured so as to mediate resistance to, and distributions of, affect. As the process of mnemic re-polarization takes place, we can learn much by examining the particulars of the process. It is to be noted that although even one or two regressions are therapeutically demonstrable in the amelioration of pathology to some substantial degree, 30 or more regressions are needed for the full effect to become solid. A full mnemic re-consolidation then, simply by looking at those familiar numbers, is likely a function of hippocampal mnemic instantiation, and subsequent hippocampal-neocortical consolidation ([7], p. 157) [29]. The effect of such a complete mnemic reconsolidation, is to alter the non-commutative template structure. Once altered, the pathogenic contributors are reduced to lower levels of less reactivity in distributional and repressive mediation, and, the new memories are allotted a predominant place. The basic circuit balance is thereby altered. So in re-polarization, effective pathological amelioration is accomplished by way of therapeutically inculcated variance in non-commutative topography via regression, via dynamic hippopcampal plasticity, and then, neocortical consolidation. Clearly, hippocampal plasticity and mnemic functioning are key to the secondary neocortical consolidation, and, demonstrated plasticity is insufficient to gain ground with less than an unrealistic number of difficult regressive therapeutic interventions. 
We wish to suggest a new method whereby the "experiential template” may be topographically re-defined and pathology lessened, without need for direct regressions, drugs, DBS or the direct access to unconscious repressed material. A process of substitutive mnemic alteration by partial regressive augmentation is implied. This may well allow some great measure of healing, by closely approaching the cause.

\subsection{Affective Regulatory Circuitry Analysis}

Schore has discovered two circuits which are primary in development, and function in opposition to each other: the dopaminergically modulated sympathetic ventral tegmental limbic circuit, and the noradrenergically modulated lateral parasympathetic tegmental limbic circuit [Schore as cited in [10], pp. 234-235]. The sympathetic circuit, which we propose underlies intersubjective Alpha Function [11] [12] [30] is formed, much as Bion had supposed, as a function of the dyadic exchange between infant and mother of glance and gaze, and we will add an inference which is quite obvious and easily supported ([7], p. 272) [31] [32] as infants engaged in the exchange of maternal glances are usually being held, that maternal touch and the subsequent addition of neuropeptides/endorphins also have a part to play in creating the result:

"It is hypothesized that maternal regulated high intensity socioaffective stimulation provided in the ontogenetic niche, specifically occurring in dyadic psychobiologically attuned, arousal amplifying, face to face reciprocal gaze transactions, generates and sustains positive affect in the dyad. These transactions induce particular neuroendocrine changes which facilitate the expansive innervation of deep sights in orbitofrontal areas, especially in the early maturing visuospatial right hemisphere, of ascending subcortical axons of a neurochemical circuit of the limbic system - the sympathetic ventral tegmental limbic circuit.” (Schore as cited in [10], p. 234).

The famous studies from the 1940's conducted by Spitz ([7], p. 262) [33] may well imply the primacy of this developmentally innervated brain circuitry extends to include the most basic dependence: that of life itself. Specifically: if deprived of maternal touch and gaze, the infant may well die. The sympathetic tegmental limbic circuit is dopaminergically modulated, and can rightly be thought of as a primary manifestation of libidinal excitation and discharge ([10], p. 237). It should be noted that the dopaminergic and opioid systems and circuitry which respond to create the good feelings which reinforce socially mediated behavior, both involve many of the same areas, such as the ventral tegmental area, where the A-10 mesolimbic dopamine cells are located ([7], p. 118). Neuropeptides such as the endogenous opioids including beta-endorphin which is triggered by social cues and touch, have a primary role in creating social bonds, quelling pain, both physical and mental, are key in alleviating separation distress, creating sexual reward, and addictive reinforcement ([7], pp. 255, 264). So we can see here, in the formation of the sympathetic ventral limbic circuit triggered by maternal exchanges of glance, sight and touch, a source of libido, an energetic dopaminergic circuit which up-mediates arousal and shapes behavior, formed presumably by way of allocating both endorphins, and those neuroendocrine functions involved with encouraging the substantial innervations of dopaminergic projections into orbitofrontal areas. Here, in the activity of the completed circuit, along with the peptide systems, dopamine and opioids serve their reward and motivational functions as social and energetic contributors.

The contrary circuit, the parasympathetic lateral limbic circuit, is to be thought of as a balance, a cut off, a competing inhibitory system to counter the rewarding energetic expression of the sympathetic circuit ([10], p. 237). This circuit functions to stop our energetic libidinal expression: functional, conditional, affect regulation in response to social cues (ibid. pp. 234-238) and so, can best be understood as the physiological structure triggered by social disapproval: by shame and guilt. Both of these circuits are innervated into the orbitofrontal areas, which mediate social cues and functioning, just as one would expect.

As we have stated, we propose improper excessive repressive/parasympathetic balance between these two circuits, may well have a fundamental and in some degree causative influence in creating pathology [11] [12]. Ergo: pathology may well be lessened to adjust that balance. We have established that said preponderant balance is likely the product to some substantial degree of initial dyadic interaction between mother and infant [11] [12]. We will attempt to reshape that result, via symbolic access to pertinent hippocampal attachment memory, aided by neuropeptide induced mnemic plasticity. This will change the template structure and circuit balance. First the mnemic symbolism will be articulated, then the neuropeptide and proposed method.

Why access memory with symbolism, and what would such a symbol associated with primary dopaminergic circuitry be? 


\subsection{The Alpha Function Key}

As the infant progresses through the initial 18 month period during which the sympathetic and parasympathetic limbic circuits are fully formed, the infant masters several stages of differentiation. It is now accepted through the work of Klein (1952) [34] and empirical demonstration, that a developmental/behavioral correlation at the age of four months, exists between infants categorized as attachment secure or disorganized, "dis-coordinated" [disorganized in the sense of being unable to properly integrate the intermeshed and exclusive psychical manifestations of separation RAGE and FEAR as they conflict and inhibit SEEKING and CARE] [35]. The infant at this stage singles out the mother as a separate object which is essential for CARE, and that this fact is then made evident by the manifestations of separation-RAGE and stranger-FEAR, which become manifest at 7 - 8 months of age ([35], p. 47). To observe firsthand, the interactions between mother and infant, the effect is obvious to casual observation: the mother's face is the infant's entire world, once indistinct as an object, now, once engaged in the exchange of gaze, touch and glance, only semi-distinct from himself, her face responds to his affects and anticipates as if part of himself, as if the world itself were a loving extension of the infant, a responsive and inclusive extension of himself. Here, we see the essence of empathy: identification with the world. Note that we make no mention of the less important distinction of identification with mankind, which is a small and far less important embedded sub-aspect associated with mirror neurons, a sub-aspect of this most vital and needful result, identification with the entire of the world-Empathy [11] [12]. It is this which we will substitute for the pathogenic content.

Next please recall that all the world is given its quality and definition by way of symbolism [21] [22]. Remember also, that symbolism is the mind's intra-systemic means of communication, i.e., the unconscious becomes available to consciousness once symbolized in dreams [9] (1900). The mother, has undergone much additional layering in her symbolic meaning and impression since we were 18 months old! For this reason, to imagine her face involved in the infantile exchange of maternal gaze and glance is ineffective, as she means many things now symbolically beyond the early formative impression. The solution is to craft a symbolic image which is directly resonant to the initial impression of the circuitry's formative process. To engage the circuitry, the following symbolic image must be formed in the mind's eye, and, entered into as if a dream. The result is a peaceful, safe, content state indicative of beta endorphin activity, created by manually engaging the formative aspects of the sympathetic circuitry which connects the orbitofrontal areas with the limbic. We should observe that as beta endorphin activity is increased, pain response is decreased, and other empirically testable responses will be found, like a characteristic EEG associated with secure low stress states, rather than the easily defined activity of mentation associated with fear, and anxious stress. Here is the symbolic key, symbolized from the source formative impression which caused initial innervation, that in turn, activates the circuitry. [The first paragraph is hypnotic introduction, the image is below. This is abbreviated. Alternative methods are also proposed below in the discussion section]:

"Please relax, hear, the water, and listen to my voice. There is a meadow, within the forest, surrounded by trees, stirring, a distant breeze. The day is still and quiet. Listen to the shining brook, spilling itself, over smooth rock and sand, listen, as it splashes, so near the meadow.

The sun is pouring down upon your face, shining and warm, golden and loving is this light, a light you are folded into, and have created, shining, pouring back up into the arch of heaven, spilling up from your glad face, and again down to fill you, the trees nodding as you dream them, the sky golden and warm as you have poured it — and back around — for it has dreamt you...now as the world, of the world, nourished and warmed, the circle complete, a round of golden warmth and light, spilling into the world and returning again, unto you, and again, you unto it...and all the world is eternal, safe, nourished and nourishing, a circle of happiness, pouring down and returning, warm and sweet, the circle glowing, life spilled round into warmth—and golden light."

We will note at this point that this approach in the context of re-polarization has proven highly effective, and add that the image may be associated with a piece of music, and that this music once brought to mind, creates the effect as easily as the image. We will clearly admit that it may be possible to use the unsymbolized image, although we believe it is unlikely.

\subsection{Safe Effective Hippocampal Plasticity Induced via Neuropeptide}

Oxytocin has been demonstrated to induce hippocampal plasticity and augment social bonding [7] [8] [36]-[39]. Clearly, it is a perfect fit for our needs. We will attempt to use a sort of resonant semi-regressive effect, to add 
cathexis to the substitute ideation, and raise its status and the resultant affective contributions to the transference structure mediated by the non-commutative experiential template. All we need do is create mnemic plasticity (and unconscious access), and input the appropriate cathexis. Variations are discussed below the following basic outline.

1) The patient is given Oxytocin, and perhaps a light dose of hypnotic sedative or other compound to encourage a hypnoid state.

2) Once positioned in a reclined posture under dim light with eyes closed, the image is (hypnotically) presented, perhaps along with a piece of favored music.

3) The patient is instructed to recall the image and music, and keep the image and music in the back of their mind at all times. The patient is instructed to invigorate the image in their mind under conditions of stress, as a coping strategy.

4) The therapy is repeated as often as needed to add new layers of increased cathexis to the formative maternal attachment dynamic.

5) This should increase the coveted psychical contributions of alpha function, through reorientation of the pathogenic topography of the ego itself, by substituting healthy unconscious dynamics in the non-commutative template hierarchy for unhealthy contributions forming the transference: alteration of the basic sympathetic/ parasympathetic limbic/orbitofrontal circuitry balance toward dopaminergic predominance.

\subsection{Predictions}

1) Although it is not necessary for the technique to be effective, we predict that if tested, this method will reveal specific pieces of epigenetic (unconscious/phylogenetic) information to be suppressed by way of this procedure, and others expressed [25]-[28]. Once identified using the methods just referenced or some other, epigenetic analysis will reveal altered suppressions and expressions by way of histone code variance and chromatin/heterochromatin proportional analysis: Methylation of lysines H3K4 and H3K36 is correlated with transcriptional activation while demethylation of H3K4 is correlated with silencing of the genomic region. Methylation of lysines H3K9 and H3K27 is correlated with transcriptional repression. Particularly, H3K9me3 is highly correlated with constitutive heterochromatin.

2) We predict that this method will not require the addition of toxic drugs in the usual high doses, as the persistence and presentation of memory are the efficacious therapeutic mechanisms.

3) We predict in cases of LRRK2 mutation, this therapy may well have a prophylactic effect in preventing, or perhaps delaying, the onset of active Parkinsonian symptoms.

\subsection{Condensation of Major Points}

1) Sympathetic/parasympathetic dopaminergic/noradrenergic balance between opposing basic affective regulatory circuitry associated with the "SEEKING system", guilt and repression, may well be an unacknowledged causal contributing element in diverse pathologies such as depression, OCD, and PD: This "parasympathetic pathological genesis" is the parasympathetic basis of pathology to be curatively altered.

2) Conductance levels, targets and approaches for DBS should not disturb (and/or may reestablish) homeostasis and coordinated intrasystemic functioning. This is the principle of homeostatic conductance.

3) Re-entrainment of the pathologically degenerative SN rhythmic output in PD via DBS, to the rhythm of the unaffected VTA (or mutual SN/VTA electro-rhythmic re-entrainment) is predicted to ameliorate pathology.

4) Efferent projections from the nucleus accumbens-septi to the SN may account for predicted improvement in PD and other pathologies over time when DBS is applied to the superolateral branch of the medial forebrain bundle under conditions of homeostatic conductance.

5) a) Low level septal stimulation in keeping with homeostatic conductance may improve PD and other pathology; b) Delivery of neurotensin or kappa blockers may improve PD and other pathology.

6) Hippocampal plastic mnemic therapy may ameliorate the diverse pathologies specified, by way of internal mnemic restructuralization and subsequent up-mediation of coordinated dopaminergic and endogenous opioid distributions without necessitating recourse to surgery or toxic drugs.

\subsection{Variations, Questions and Discussion Points}

DBS sections: 
a) For OCD, the ventral capsule/ventral striatum is the typical DBS target. It is known that the ventral capsule/ ventral striatum are also stimulated from DBS applied to the superolateral branch of the medial forebrain bundle [40].

How long will it take for a low level homeostatic approach to take effect, and how effective will it be?

b) Studies have demonstrated extensive dopaminergic systemic destruction yielding depressive symptomatology in rodents, can be ameliorated via bilateral MFB DBS [40] [41]. Can partial dopaminergic necrosis be rescued with homeostatic DBS...and to what degree?

c) Is it possible to establish a baseline subject specific metric for sympathetic/parasympathetic limbic/orbitofrontal balance [12], and hence, establish a primary pathological conditional assessment, by virtue of which clinical improvement can be directly assessed?

d) Some studies seem to indicate that chronic DBS may have little deleterious effect [41]. Is there justification for the notion of homeostatic conductance?

Hippocampal plastic mnemic therapy section:

a) It is possible that an unsymbolized version of the image will be more effective.

b) It is possible that a surrogate therapist may provide physical contact to augment the process to greater effect in some cases.

c) It is possible that this approach could help schizophrenics. If maternal inconsistency is at the fount of schizophrenia, patients may be aided in intensive therapy. Schizophrenia appears in some studies to be responsive to Oxytocin [42]. An entirely new attachment structure may be created.

d) Other images each detailed to meet the specific maternal and paternal infantile needs of the particular patient may be used.

e) Other images of maternal interactivity resonant with the innervation of the primary dopaminergic sympathetic circuitry can be derived.

f) Default Mode Network (ego) [24] destructuralization and desensitization are associated with meditative practice [43]. Images or other means such as sound or visual imagery extracted from traditional meditative practice may prove via careful empirical study and experiment to restructure ego when extracted and applied thusly, and so improve neurotic, depressive and other symptomatology.

g) Head gear which uses sound and visual imagery may be effective.

h) Full regressions used to augment re-polarization proper may possibly be encouraged with the use of 5-HT blocking agents, or other psychoactive compounds if closely supervised.

i) Additive cathexis associated with therapeutically derived archetypal structure may be augmented [44].

j) In the case of "h" above, secondary therapeutic mnemic content may counteract pathology associated with neurotic super-ego at the source level.

\section{Conclusion}

New approaches based on the trustworthy disciplines of Affective Neuroscience and Neuropsychoanalysis are worthy of serious exploration. Homeostatic conductance may offer us a guideline for new directions and strategies using DBS for OCD, depression and Parkinson's. Reorientation of the basic affective regulatory circuitry in man, through chemical, electro-stimulatory and new noninvasive means, may offer productive avenues of approach yielding pro-dopaminergic adjustment of a heretofore unacknowledged causal element in pathology: Limbic/Orbitofrontal parasympathetic activational predominance in basic affective circuitry. To address this neglected element may provide new fruitful directions toward the treatment of a variety of somatic, psychological and interrelated pathologies.

\section{References}

[1] Perestelo-Perez, L., Rivero-Santana, A., Perez-Ramos, J., Serrano-Perez, P., Panetta, J. and Hilarion, P. (2014) Deep Brain Stimulation in Parkinson's Disease: Meta-analysis of Randomized Controlled Trials. Journal of Neurology/ Database of Abstracts of Reviews of Effects (DARE): Quality-Assessed Reviews, 261, 2051-2060. http://www.ncbi.nlm.nih.gov/pubmedhealth/PMH0063168/

[2] Liu, Y., Li, W., Tan, C., Liu, X., Wang, X., Gui, Y., Qin, L., Deng, F., Hu, C. and Chen, L. (2014) Meta-Analysis Comparing Deep Brain Stimulation of the Globus Pallidus and Subthalamic Nucleus to Treat Advanced Parkinson Disease. Journal of Neurosurgery, 121, 709-718. http://dx.doi.org/10.3171/2014.4.JNS131711 
[3] Weaver, F., Kenneth Follett, K., Hur, K., Dolores Ippolito, D. and Stern, M. (2012) Deep Brain Stimulation in Parkinson Disease: A Metaanalysis of Patient Outcomes. Journal of Neurosurgery, 117, 956-967.

[4] Smith, D.F. (2014) Exploratory Meta-Analysis on Deep Brain Stimulation in Treatment-resistant Depression. Acta Neuropsychiatr, 26, 382-384. http://dx.doi.org/10.1017/neu.2014.22

[5] Alonso, P., Cuadras, D., Gabriëls, L., Denys, D., Goodman, W., Greenberg, B.D., et al. (2015) Deep Brain Stimulation for Obsessive-Compulsive Disorder: A Meta-Analysis of Treatment Outcome and Predictors of Response. PLoS ONE, 10, e0133591. http://dx.doi.org/10.1371/journal.pone.0133591

[6] Kisely, S., Hall, K., Siskind, D., Frater, J., Olson, S. and Crompton, D. (2014) Deep Brain Stimulation for Obsessive-compulsive Disorder: A Systematic Review and Meta-Analysis. Psychological Medicine, 44, 3533-3542. http://dx.doi.org/10.1017/S0033291714000981

[7] Panksepp, J. (1998) Affective Neuroscience: The Foundations of Human and Animal Emotions. Oxford Press, New York.

[8] Panksepp, J. (2012) The Archaeology of Mind: Neuroevolutionary Origins of Human Emotions. W.W. Norton and Company, NY.

[9] Freud, S. (2001) The Standard Edition of the Complete Psychological Works of Sigmund Freud Volumes One Through Twenty-Four. Hogarth Press, London.

[10] Kaplan-Solms, K. and Solms, M. (2002) Clinical Studies in Neuropsychoanalysis: Introduction to a Depth Neuropsychology. Karnac Press, London.

[11] Norman, R.L. (2013) Who Fired Prometheus? The Historical Genesis and Ontology of Super-Ego and the Castration Complex: The Destructuralization and Repair of Modern Personality-An Essay in Five Parts. The Journal of Unconscious Psychology and Self-Psychoanalysis, 1, 1(a)-26. http://www.thejournalofunconsciouspsychology.com/web documents/who fired prometheus black watch.pdf

[12] Norman, R.L. (2014) Limbic Connectivity and Sympathetic Neural Balance: The Primary Psycho-Physiological Locus of Affect. Mind Magazine. http://www.mindmagazine.net/\#!new-ideas/czpl

[13] Gazzaniga, M., Ivry, R. and Mangun, G. (2009) Cognitive Neuroscience: The Biology of the Mind. Norton Press, London.

[14] Coenen, V.A., Honey, C.R., Hurwitz, T., Rahman, A.A., McMaster, J., Burgel, U. and Madler, B. (2009) Medial Forebrain Bundle Stimulation as a Pathophysiological Mechanism for Hypomania in Subthalamic Nucleus Deep Brain Stimulation for Parkinson's Disease. Neurosurgery, 64, 1106-1114; Discussion 1114-1115. http://dx.doi.org/10.1227/01.NEU.0000345631.54446.06

[15] Johnson, M.D., Miocinovic, S., McIntyre, C.C. and Vitek, J.L. (2008) Mechanisms and Targets of Deep Brain Stimulation in Movement Disorders. Neurotherapeutics: The Journal of the American Society for Experimental Neuro Therapeutics, 5, 294-308. http://dx.doi.org/10.1016/j.nurt.2008.01.010

[16] Brichta, L. and Greengard, P. (2014) Molecular Determinants of Selective Dopaminergic Vulnerability in Parkinson's Disease: An Update. Frontiers in Neuroanatomy, 8, 152. http://dx.doi.org/10.3389/fnana.2014.00152

[17] Subramaniam, M., Althof, D., Gispert, S., Schwenk, J., Auburger, G., Kulik, A., Fakler, B. and Roeper, J. (2014) Mutant $\alpha$-Synuclein Enhances Firing Frequencies in Dopamine Substantia Nigra Neurons by Oxidative Impairment of AType Potassium Channels. The Journal of Neuroscience, 34, 13586-13599. http://dx.doi.org/10.1523/JNEUROSCI.5069-13.2014

[18] Subramaniam, M., Kern, B., Vogel, S., Klose, V., Schneider, G. and Roeper, J. (2014) Selective Increase of in Vivo Firing Frequencies in DA SN Neurons after Proteasome Inhibition in the Ventral Midbrain. European Journal of Neuroscience, 40, 2898-2909. http://dx.doi.org/10.1111/ejn.12660

[19] Schlaepfer, T.E., Bewernick, B.H. and Kayser, S. (2013) Rapid Effects of Deep Brain Stimulation for TreatmentResistant Major Depression. Biological Psychiatry, 73, 1204-1212. http://dx.doi.org/10.1016/j.biopsych.2013.01.034

[20] Norman, R. (2015) (Semi)-Regressive Plastic Attachment Therapy. Mind Magazine. http://www.mindmagazine.net/\#!new-ideas/czpl

[21] Norman, R. (2015) Quantum Unconscious Pre-Space: A Psychoanalytic Neuroscientific Analysis of the Cognitive Scienceof Elio Conte-The Hard Problem of Consciousness, New Approach and Directions. Neuroquantology, 13, 487-501. http://dx.doi.org/10.14704/nq.2015.13.4.869

[22] Norman, R.L. (2013) The Quantitative Unconscious: A Psychoanalytic Perturbation-Theoretic Approach to the Complexity of Neuronal Systems in the Neuroses. The Journal of Unconscious Psychology and Self-Psychoanalysis, 1, 1(b)-19.

http://www.thejournalofunconsciouspsychology.com/web documents/the quantitative unconscious black watc h_use_pdf 
[23] Norman, R.L. (2013) Re-Polarization Theory: From Native Psychoanalysis to Sublimation-The Practical Reconstruction of Modern Personality. The Journal of Unconscious Psychology and Self-Psychoanalysis, 1, 1(c)-63. http://www.thejournalofunconsciouspsychology.com/web_documents/re-polarization_theory.pdf

[24] Carhart-Harris, R.L. and Friston, K.J. (2010) The Default-Mode, Ego-Functions and Free-Energy: A Neurobiological Account of Freudian Ideas. Brain, 133, 1265-1283. http://dx.doi.org/10.1093/brain/awq010

[25] Norman, R.L. (2015) Modern Man of Phylogeny, Guilt, Obedience and Consequence-An Answer to Old Problems. Mind Magazine. http://www.mindmagazine.net/\#!new-ideas/czpl

[26] Norman, R.L. (2015) Mnemic Psycho-Epigenetics: The Foundational Basis of Depth, Archetype and Synthesis in Psychology. Mind Magazine. http://www.mindmagazine.net/\#!new-ideas/czpl

[27] Norman, R.L. (2015) The Epigenetic Unconscious pt. 1. Mind Magazine. http://www.mindmagazine.net/\#!new-ideas/czpl

[28] Norman, R.L. (2015) The Epigenetic Unconscious pt. 2. Mind Magazine. http://www.mindmagazine.net/\#!new-ideas/czpl

[29] Stickgold, R., Hobson, J., Fosse, R. and Fosse, M. (2001) Sleep, Learning, and Dreams: Off-Line Memory Reprocessing. Science, 294, 1052-1057. http://dx.doi.org/10.1126/science.1063530

[30] Brown, L. (2011) Intersubjective Processes and the Unconscious. Routledge, London.

[31] Keveren, E.B., Martensz, N. and Tuite, B. (1989) Beta-Endorphin Concentrations in CSF of Monkeys Are Influenced by Grooming Relationships. Psychoneuroendocrinology, 14, 155-161. http://dx.doi.org/10.1016/0306-4530(89)90065-6

[32] Montagu, A. (1978) Touching: The Human Significance of the Skin. Harper and Row, New York.

[33] Bowlby, J. (1980) Attachment and Loss. Volume 1, Attachment. Basic Books, New York.

[34] Klein, M. (1952) Some Theoretical Conclusions regarding the Emotional Life of the Infant. In: The Writings of Melanie Klein, Volume 8: Envy and Gratitude and Other Works, Hogarth Press, London, 61-94.

[35] Hopkins, J. (2013) Conflict Creates an Unconscious Id. Neuropsychoanalysis, 15, 45-48. http://dx.doi.org/10.1080/15294145.2013.10773718

[36] Lin, Y., Huang, C. and Hsu, K. (2012) Oxytocin Promotes Long-Term Potentiation by Enhancing Epidermal Growth Factor Receptor-Mediated Local Translation of Protein Kinase M $\zeta$. The Journal of Neuroscience, 32, 15476-15488. http://www.jneurosci.org/content/32/44/15476.full http://dx.doi.org/10.1523/JNEUROSCI.2429-12.2012

[37] Monks, D., Lonstein, J. and Breedlove, M. (2003) Got Milk? Oxytocin Triggers Hippocampal Plasticity. Nature Neuroscience, 6, 327-328. http://dx.doi.org/10.1038/nn0403-327

[38] Leuner, B., Caponiti, J. and Gould, E. (2012) Oxytocin Stimulates Adult Neurogenesis Even under Conditions of Stress and Elevated Glucocorticoids. Hippocampus, 22, 861-868. http://dx.doi.org/10.1002/hipo.20947

[39] Garcia-Segura, L. (2009) Hormones and Brain Plasticity. Cellular and Molecular Neuroendocrinology Laboratory, Cajal Institute, Oxford University Press, Oxford. http://dx.doi.org/10.1093/acprof:oso/9780195326611.001.0001

[40] Furlanetti, L.L., Coenen, V.A. and Döbrössy, M.D. (2016) Ventral Tegmental Area Dopaminergic Lesion-Induced Depressive Phenotype in the Rat Is Reversed by Deep Brain Stimulation of the Medial Forebrain Bundle. Behavioral Brain Research, 299, 132-140. http://dx.doi.org/10.1016/j.bbr.2015.11.036

[41] Furlanetti, L.L., Coenen, V.A., Aranda, I.A. and Döbrössy, M.D. (2015) Chronic Deep Brain Stimulation of the Medial Forebrain Bundle Reverses Depressive-Like Behavior in a Hemiparkinsonian Rodent Model. Experimental Brain Research, 233, 3073-3085. http://dx.doi.org/10.1007/s00221-015-4375-9

[42] MacDonald, K. and Feifel, D. (2012) Oxytocin in Schizophrenia: A Review of Evidence for Its Therapeutic Effect. Acta Neuropsychiatrica, 24, 3130-3146. http://dx.doi.org/10.1111/j.1601-5215.2011.00634.x

[43] Brewer, J., Worhunski, P., Gray, J., Tang, Y., Weber, J. and Kober, H. (2011) Meditation Experience Is Associated with Differences in Default Mode Network Activity and Connectivity. PNAS, 108, 20254-20259. http://www.pnas.org/content/108/50/20254.full http://dx.doi.org/10.1073/pnas.1112029108

[44] Norman, R.L. (2015) Object, Archetype, and the Social Myth of Conditional Regard: The Applied Neuroscience of Social Healing. Mind Magazine. http://www.mindmagazine.net/\#!new-ideas/czpl 